\title{
Modelling the Radiolysis of Silver Nitrate Solutions in presence of Bromide Ions in Liquid-Phase Transmission Electron Microscopy
}

\author{
$\underline{\text { Mehran Taherkhani }}{ }^{1}$, Birk Fritsch ${ }^{1}$, Michael P. M. Jank ${ }^{2}$, Erdmann Spiecker ${ }^{3}$, Andreas Hutzler ${ }^{1,4}$ \\ ${ }^{1}$ Electron Devices (LEB), Friedrich-Alexander University Erlangen-Nürnberg (FAU), Erlangen, \\ Germany. \\ ${ }^{2}$ Fraunhofer Institute for Integrated Systems and Device Technology IISB, Erlangen, Germany. \\ ${ }^{3}$ Institute of Micro- and Nanostructure Research (IMN) \& Center for Nanoanalysis and Electron \\ Microscopy (CENEM), FAU, Erlangen, Germany. \\ ${ }^{4}$ Helmholtz Institute Erlangen-Nürnberg for Renewable Energy (IEK-11), Forschungszentrum Jülich \\ GmbH, Erlangen, Germany.
}

In this study, we model the radiolysis chemistry of aerated $\mathrm{AgNO}_{3}$ solutions during liquid-phase transmission electron microscopy (LP-TEM) in presence of bromide ions using a Python-based adaption of the radiolysis model of Schneider et al. [1]. We verify the model qualitatively by comparing the results with experiments using a graphene-supported microwell liquid cell [2,3]. LP-TEM is a powerful, yet novel method for analyzing dynamic processes of nanostructures in liquid environment. One of the major challenges for enabling LP-TEM to become a standard characterization method, however, is the development of accurate physical models describing electron-beam interactions with liquid specimen, e.g. heating [4], radiolysis [1,2,5,6], and charging effects [7]. In particular, the model of Schneider et al. [1] describing radiolysis of deionized and deaerated water under electron beam irradiation has been used intensively to explain a vast variety of LP-TEM-specific phenomena.

Recently, complex particle growth and dissolution studies investigating gold-silver heterostructures obtained high interest in research [2,8]. In order to support the proposed interpretation of experimental results, we elaborate an adaption of Schneider's radiolysis script to describe the reaction chemistry of aerated $\mathrm{AgNO}_{3}$ solutions in presence of bromide ions, which originate from stabilizing agents like cetrimonium bromide (CTAB). Besides water, this requires 36 additional species and yields a reaction set comprising 232 coupled ordinary differential equations. The original Matlab ${ }^{\odot}$-based script is ported to open source software (Python) as a first step towards an easily accessible simulation program. We find excellent agreement in both performance and outcome of our script with the initial version. A validation of our model is achieved by comparison with LP-TEM experiments on silver-shell growth on gold nanorods using an in-house developed liquid-cell architecture [2,3] in combination with a Philips CM-30 (S)TEM operated at $300 \mathrm{kV}$ and electron flux densities between $10^{4}$ and $10^{5} \mathrm{e}^{-} \mathrm{nm}^{-2} \mathrm{~s}^{-1}$.

Figure 1a shows the outcome of the simulated model describing the evolution of concentrations of reactants at different $\mathrm{AgNO}_{3}$ concentrations (10-2 $\mathrm{M}$ : solid lines; $10^{-3} \mathrm{M}$ : dashed lines). A comparison of both simulated concentrations reveals main differences for an excess of $\mathrm{Ag}^{+}$, suggesting an equilibrium balance between precipitated $\mathrm{AgBr}$ and $\mathrm{Ag}^{+}$ions required for formation of elementary silver (black data). The concentration of $\mathrm{AgBr}$ (blue data) remains nearly constant at an excess of $\mathrm{Ag}^{+}\left(10^{-2} \mathrm{M}\right)$ in contrast to the low concentration $\left(10^{-3} \mathrm{M}\right)$ where it drops by more than $50 \%$. Figure $1 \mathrm{~b}$ shows the dependency of steady state concentrations on the dose rate for different silver species. Previous LP-TEM experiments at high dose rates presented by Hutzler et al. [2] revealed underpotential deposition of silver shells on Au nanorods. This observation correlates with the layer-by-layer dissolution of AgBr nanocrystals, controlled by the electron flux density via distinctly generated radiolytic species. These findings are in good agreement with the decrease of steady state concentrations of $\mathrm{Ag}^{+}$(Fig. 1b, red data) due to a reduction to 
$\mathrm{Ag}$ (black data) on the surface of gold nanorods. This is counterintuitive due to $\mathrm{AgBr}$ being highly sensitive to radiation. A disruption of the solution equilibrium of $\mathrm{AgBr}$ and its solvated ion pair (Fig. 1a, $\mathrm{Br}^{-}$: green data and $\mathrm{Ag}^{+}$: red data) for low $\mathrm{Ag}^{+}$concentrations and the presence of high concentrations of hydroxide radicals (which are able to compensate free charge carriers in $\mathrm{AgBr}$ ) are assumed to be the driving force for this anomalous behavior. Only at high dose rates, layer-by-layer dissolution of $\mathrm{AgBr}$ is observed, which supports this hypothesis. At low dose rates, in turn, the expected growth of silver nanoparticles by direct reduction of $\mathrm{AgBr}$ is observable.

In summary, we present a comprehensive kinetic model for simulating radiolysis of aqueous solutions containing $\mathrm{AgNO}_{3}$ and bromide ions. The outcomes are in good agreement with experimental data. It is, thus, a useful tool for further investigations of the chemical and physical observations happening during LP-TEM.

[1] N M Schneider et al, J. Phys. Chem. C 118 (2014), p. 22373.

[2] A Hutzler et al, Nano Lett. 18 (2018), p. 7222.

[3] A Hutzler et al, J. Vis. Exp. (2019), Art. No. e59751.

[4] B Fritsch et al, Nanoscale Adv. 3 (2021), p. 2466.

[5] J M Grogan et al, Nano Lett. 14 (2014), p. 359.

[6] A Hutzler et al, Adv. Mater Interfaces 6 (2019), Art. No. 1901027.

[7] P Liu et al, Nano Lett. 20 (2020), p. 1944.

[8] K Aliyah et al, J. Phys. Chem. Lett. 11 (2020), p. 2830.

[9] Financial support by the German Research Foundation (DFG) via the Cluster of Excellence "Engineering of Advanced Materials (EAM)" as well as the Research Training Group GRK 1896 "In situ microscopy with electrons, X-rays and scanning probes" is gratefully acknowledged.
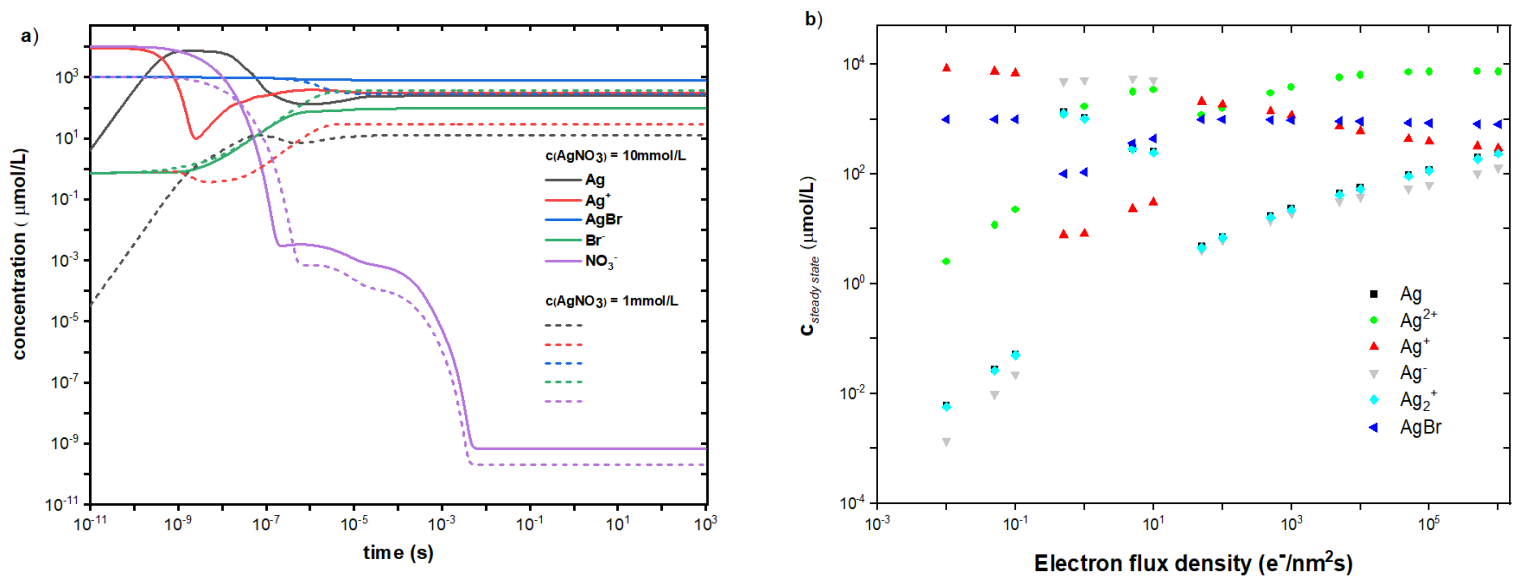

Figure 1. Simulation results of (a) the evolution of the concentration of distinct species emerging during LP-TEM for $\mathrm{AgNO}_{3}\left(10^{-2} \mathrm{M}\right.$ : solid lines; $10^{-3} \mathrm{M}$ : dashed lines) and $\mathrm{Br}^{-}$containing solutions (CTAB, $\mathrm{c}=10^{-3} \mathrm{M}$ ) at the electron flux density of $10^{5} \mathrm{e}^{-} \mathrm{nm}^{-2} \mathrm{~s}^{-1}$ and (b) dependency of the steady state concentrations of relevant silver species on the electron flux density. 